\title{
How do chaperonins fold protein?
}

\author{
Fumihiro Motojima ${ }^{1}$ \\ ${ }^{1}$ Department of Molecular Biosciences, Faculty of Life Sciences, Kyoto Sangyo University, Kamigamo-Motoyama, Kita-ku, Kyoto \\ 603-8555, Japan
}

Received August 22, 2014; accepted December 23, 2014

\begin{abstract}
Protein folding is a biological process that is essential for the proper functioning of proteins in all living organisms. In cells, many proteins require the assistance of molecular chaperones for their folding. Chaperonins belong to a class of molecular chaperones that have been extensively studied. However, the mechanism by which a chaperonin mediates the folding of proteins is still controversial. Denatured proteins are folded in the closed chaperonin cage, leading to the assumption that denatured proteins are completely encapsulated inside the chaperonin cage. In contrast to the assumption, we recently found that denatured protein interacts with hydrophobic residues at the subunit interfaces of the chaperonin, and partially protrude out of the cage. In this review, we will explain our recent results and introduce our model for the mechanism by which chaperonins accelerate protein folding, in view of recent findings.
\end{abstract}

Key words: molecular chaperone, protein folding, GroEL, hydrophobic interaction

\section{Molecular chaperones are necessary for protein folding in cells}

For most proteins to perform their functions in cells, they should form their native structures, which are coded in their amino acid sequences. Thus, the formation of protein structure (protein folding) is an essential process in all living organisms. The structure of native proteins depends on the minimum free energy, which is determined by a balance between the decrease in free energy (ascribed to the forma-

Corresponding author: Fumihiro Motojima, Department of Molecular Biosciences, Faculty of Life Sciences, Kyoto Sangyo University, Kamigamo-Motoyama, Kita-ku, Kyoto 603-8555, Japan.

e-mail: fmotojim@gmail.com tion of hydrogen bonding, electrostatic interactions, van der Waals interactions, and hydrophobic interactions) and the increase in free energy (due to the decrease in conformational entropy upon folding). As a result, most proteins have low (marginal) stability and are easily destabilized by small deviations in temperature or $\mathrm{pH}$ from the native condition, or by spontaneous denaturation in conformational equilibrium. As Charles Anfinsen has shown [1], in some cases, proteins can spontaneously fold from an unfolded (denatured) state into their native structure in vitro. However, for many proteins, it is practically difficult to fold spontaneously, since the irreversible protein aggregate formed by the interactions between long-lived denatured proteins results in the low yield of spontaneous folding. This becomes more problematic in cells where unfolded proteins are constantly produced by ribosomes as nascent proteins.

Molecular chaperones were first discovered as proteins that were expressed upon heat shock and were thus named as heat shock proteins (HSPs) [2]. Although the function of HSPs had been unknown for a while, in the late 1980s, it was demonstrated that many proteins require the assistance of molecular chaperones to fold into their native states in vivo and in vitro [3-6]. Molecular chaperones are categorized into several classes, each of which has a distinct amino acid sequence and tertiary structure. Major molecular chaperones are chaperonins and the Hsp70 chaperone system. The Hsp70 chaperone system consists of Hsp70, Hsp40, and nucleotide exchange factors, and facilitates the folding of denatured protein in the ATP hydrolysis-dependent reaction cycle [7]. The affinity of denatured protein to Hsp70 becomes weak in the ATP-bound state and strong in the ADP-bound state. Both Hsp70s and Hsp40s recognize short hydrophobic peptide region in denatured protein [8]. The detailed molecular mechanism by which the Hsp70 system assists in protein folding is yet unclear. 

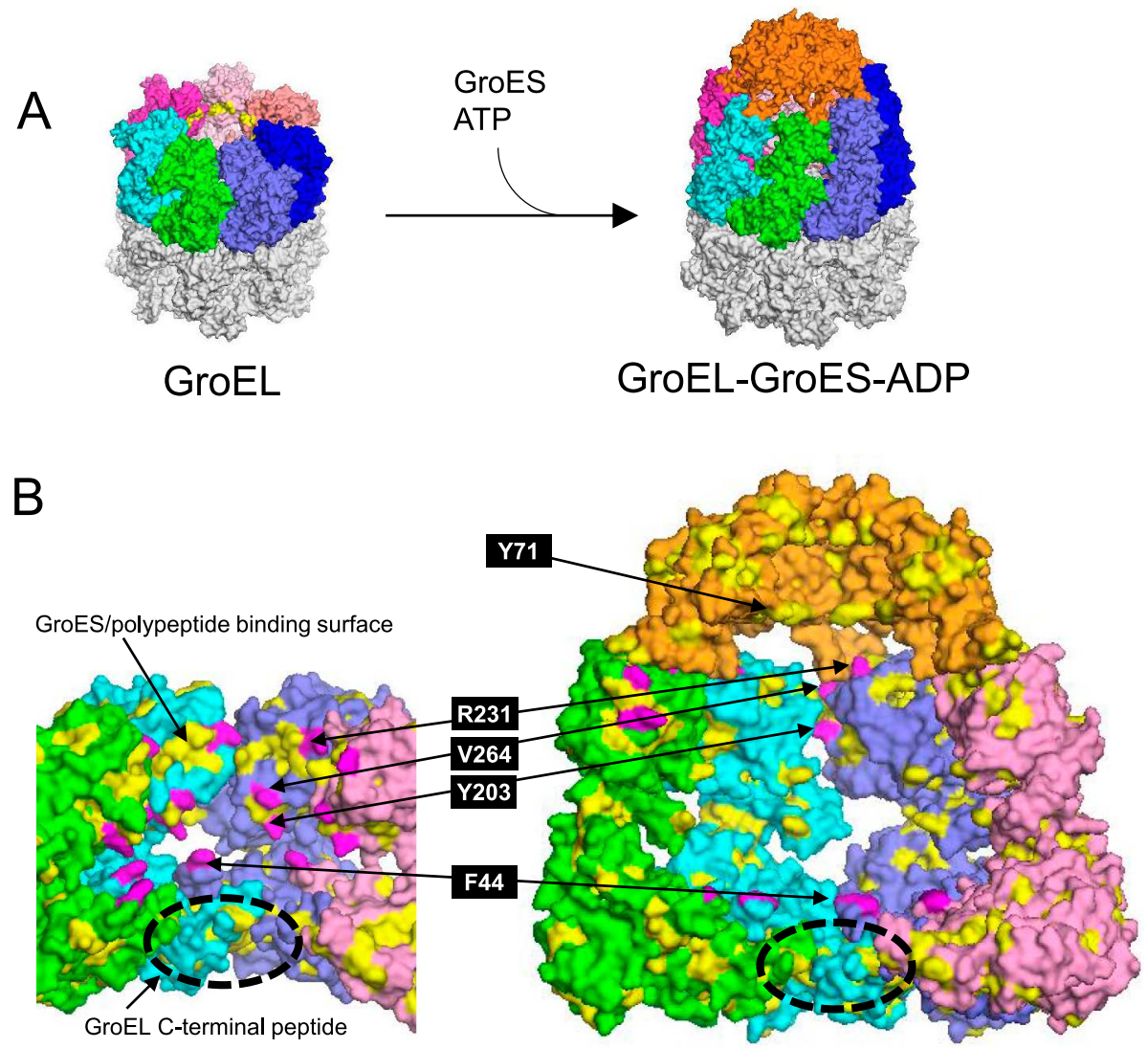

Figure 1 The structure of chaperonin. (A) The X-ray crystal structures of GroEL (left, PDB:1OEL) [9] and GroEL-GroES-ADP complex (right, PDB:1AOL) [11]. GroEL subunits in the upper heptamer ring are shown in various colors. The lower GroEL ring is colored in gray. GroES is colored in orange. (B) The inside surface of GroEL (left) and GroEL-GroES-ADP complex (right, the chaperonin cage). GroEL and GroES are colored as Fig. 1. Hydrophobic residues are colored in yellow. The residues forming disulfide bonds with denatured rhodanese are colored in magenta and labeled. Putative positions of hydrophobic C-terminal peptides of GroEL, which are not observed in the crystal structure, are shown in a black dotted circle.

Chaperonins form a double ring structure stacked backto-back, and assist protein folding in the central cavities (Fig. 1A). The class of chaperonins are subdivided into two groups. Group I chaperonins are found in bacteria as well as organelles of endosymbiotic origin, mitochondria and chloroplasts, and consist of Hsp60 and its co-chaperone Hsp10. Hsp10 associates with Hsp60 to cap the cavity of Hsp60 to form the closed chaperonin cage in which denatured protein folds. In many cases, Hsp60 and Hsp10 are homo-oligomer. Group II chaperonins are found in archaea and the cytosol of eukaryotes. Although Hsp10-type co-chaperones have not identified for group II chaperonins, the subunits of group II chaperonins contain "lid" domain to close their cavity instead. Archaeal chaperonins are composed of several different subunits and form eight- or nine-membered heterooligomeric rings. Eukaryotic chaperonins consist of eight different subunits. One of the most investigated molecular chaperones is the chaperonin GroEL/GroES from E. coli, which is essential for bacterial viability. The in vitro demonstration that the chaperonin greatly improves the folding of Rubisco, a key enzyme in carbon-fixation from photosyn- thetic bacteria, was a pioneering study elucidating the folding mechanism of chaperonins [4].

The chaperonin GroEL consists of two rings stacked back-to-back, and each ring contains seven GroEL subunits (Fig. 1A). Hydrophobic residues, which interact with a denatured protein and GroES, are aligned on the inner rim of the central cavity of GroEL $[9,10]$. GroES, a lid of the cavity, has seven mobile loops that interact with the hydrophobic surfaces of GroEL. Upon ATP binding to GroEL, GroEL binds GroES and undergoes conformational changes to form a large hydrophilic cavity (the chaperonin cage) in which a denatured protein is encapsulated [11] (Fig. 1B). In the absence of the denatured protein, either one of the two rings of GroEL binds to GroES [12]. However, in the presence of excess denatured protein, both rings of the chaperonin bind to GroES and assist in the folding of the denatured protein equivalently [13-17]. Chaperonins greatly improve the yield of protein folding, especially for stringent substrate proteins that tend to form aggregates during spontaneous folding, such as Rubisco and rhodanese, a mitochondrial protein that detoxifies cyanide $[4,18]$. Aggregate-forming interactions 

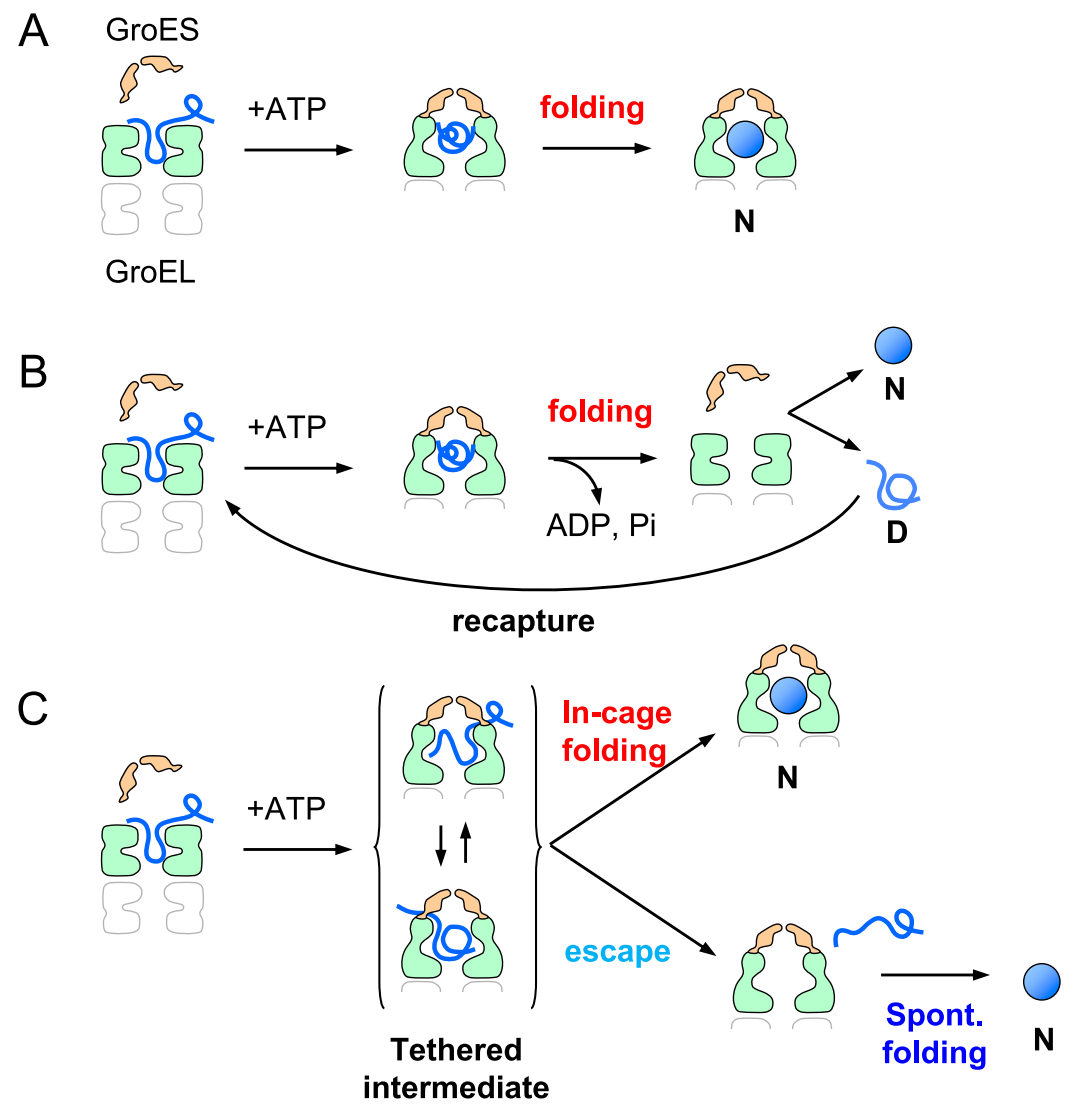

Figure 2 Schematic model of chaperonin-mediated folding. The upper rings of GroEL and GroES are colored in green and orange, respectively. Native protein $(\mathrm{N})$ and denatured protein (D) are shown as a blue circle and blue curves, respectively. (A) Passive cage model and confinement model. The passive cage model assumes that the denatured protein folds in a spontaneous manner. The confinement model assumes that space restriction in the chaperonin cage accelerates protein folding. (B) Iterative annealing model. The denatured protein is unfolded upon binding to GroEL and GroES binding. The iterative action of binding and release of the denatured protein is assumed to accelerate protein folding. (C) Tethering model. The denatured protein encapsulated inside the chaperonin cage is not freely isolated therein, but still interacts with the hydrophobic residues of the chaperonin cage, forming a tethered intermediate. The hydrophobic interaction is weak enough to allow conformational changes in the denatured protein. The denatured protein folds in the chaperonin cage (in-cage folding) or escapes out of the chaperonin cage, followed by spontaneous folding (out-of-cage folding).

among the denatured proteins are prevented through the physical isolation of the denatured protein in the chaperonin cage. Based on these observations, it was deduced that the encapsulated denatured protein freely folds in the chaperonin cage (Anfinsen (passive) cage model, Fig. 2A).

In addition to this passive folding mechanism, two active folding mechanisms have also been proposed. The iterative repetition of the binding of denatured proteins to GroEL and their release from GroEL, synchronized with ATP hydrolysis, has been proposed to accelerate protein folding (iterative annealing model, Fig. 2B). It has been proposed that protein folding is accelerated in the chaperonin cage by the space restriction therein (confinement model, Fig. 2A). Recently, it was also suggested that the structured water molecules induced by the negatively charged residues of the chaperonin cage accelerate protein folding [19].

In contrast to the former assumption that the denatured protein is completely encapsulated inside the chaperonin cage and is repulsive to the cage, we recently found that the denatured protein is not completely encapsulated inside the chaperonin cage, but interacts with hydrophobic residues at the subunit interface of the chaperonin (Fig. 1B). Moreover, the denatured protein partially protrudes out of the chaperonin cage and, in some cases, escapes spontaneously out of the cage. To explain these results, we proposed a new model, whereby the hydrophobic interaction between the denatured protein and GroEL induces the stretching of the denatured protein, changes the pathway of protein folding, and, in some cases, accelerates folding (Tethering model, Fig. 2C) [20]. In this review, we will explain our recent results and introduce our model for the mechanism by which the chaperonin accelerates protein folding. 


\section{The interaction between denatured proteins and the chaperonin cage}

Upon GroES and ATP binding to the complex formed between GroEL and the denatured protein, the denatured protein is encapsulated inside the chaperonin cage and the protein folding reaction starts. After ATP molecules are hydrolyzed to ADP in $\sim 10 \mathrm{~s}$, GroES and the encapsulated protein are released from the chaperonin cage. The iterative GroES binding and release from GroEL, coupled with ATP hydrolysis, complicates the analysis of the folding reaction in the chaperonin cage. To avoid this complication, a single-ring chaperonin mutant of SR1, which undergoes single-round protein folding without the release of GroES, has been used (Fig. 2A) [21]. Before our experiments, it was considered that all denatured proteins bound to GroEL and SR1 are encapsulated inside the chaperonin cage. We confirmed that the entire denatured protein is encapsulated inside the chaperonin cage upon GroES binding, but we further found that after encapsulation, a fraction of the denatured protein escapes from the closed chaperonin cage (Fig. 2C) [20]. Therefore, the previous report that about half of the folded green fluorescence protein (GFP) appears outside of the SR1 cage [22] is now interpreted as a result of the spontaneously folding of escaped GFP in the medium [20]. The fraction of escape is dependent on the substrate protein. About $90 \%$ of the double mutant of maltose binding protein (DMMBP, $\mathrm{MBP}(\mathrm{V} 8 \mathrm{G} / \mathrm{Y} 283 \mathrm{D}))$ escaped from the chaperonin cage, $15 \%$ of rhodanese escaped from the cage, but Rubisco hardly escaped $[20,23]$. Proteases, antibodies against substrate proteins, or the highly hydrophobic GroEL mutant N265A, added to the outside medium, inhibit the protein folding inside the SR1 cage, supporting that the denatured protein protrudes out of the chaperonin cage at least transiently [20]. Although the protrusion and escape of the denatured protein have not been previously observed, the chaperonin cage indeed has many windows, the diameters of which range from 10 to $20 \AA$ (Fig. 1B). These results imply that the conformation of the denatured protein in the cage is stretched rather than compacted.

The protrusion site of the denatured protein encapsulated inside the chaperonin cage was searched by detecting the formation of disulfide bonds between cysteine residues introduced to GroEL and those contained in denatured rhodanese [20]. In the absence of ATP, denatured rhodanese forms disulfide bonds with F44C, Y203C, R231C, and V264C exposed inside the chaperonin cage (Fig. 1B, left). These disulfide bonds are formed even after large conformational changes in GroEL upon GroES/ATP binding. Y203C and V264C are moved to the hydrophobic interface between GroEL subunits and become less exposed inside the chaperonin cage (Fig. 1B, right). R231C is moved upward and is located beneath the GroES/polypeptide binding surface. The outward movement of these hydrophobic surfaces upon GroES binding could induce the stretching of the denatured protein as suggested in the iterative annealing model. Since the denatured protein binds to antibodies against the denatured protein outside of the cage at any moment in the chaperonin-mediated folding, it is suggested that the denatured protein always interacts with these hydrophobic residues in the chaperonin cage and protrudes out of the cage (tethered intermediate, Fig 2C) [20]. The interaction of the denatured protein with dispersed hydrophobic residues in the chaperonin cage would stretch the denatured protein partially and allow the free stretched region to form partial (segmental) structures (Fig. 3). This stretching effect would not induce extensive unfolding and amide proton exchange of the denatured protein, as observed in Rubisco, DHFR, and MDH [24-26]. The hydrophobic GroES/polypeptide binding surfaces of three GroEL subunits could be reserved for the binding of the denatured protein, since GroEL or GroES oligomers containing up to three binding-defective subunits, whose residues interacting with the GroES/polypeptide binding surface are mutated, can mediate protein folding $[27,28]$. We also found that the SR1(Y203) mutant allows more escape of the denatured rhodanese out of the chaperonin cage, and folds rhodanese more slowly than SR1 does, indicating that hydrophobic interfaces are important for both the retention of the denatured protein inside the cage and folding acceleration.

\section{The measurement of the "real" in-cage folding}

The above-mentioned results also showed that the elimination of spontaneous folding outside the chaperonin cage is necessary to measure the chaperonin-mediated folding inside the cage (in-cage folding). This point has not been noticed in previous studies but is especially important for the correct comparison between chaperonin-mediated folding and spontaneous folding. To measure in-cage folding exclusively, trapGroEL(D87K), a mutant of GroEL which binds denatured proteins but cannot mediate folding [10], was added to the folding reaction solution. We also found that guanidium chloride, which was often used to denature substrate proteins, induces artifacts that increase in the escape of denatured proteins out of the cage and destabilize GroES binding to SR1, which enables SR1 to fold substrate proteins in an iterative manner as GroEL does [23].

In several reports, guanidium chloride was used without considering the iterative folding cycle of SR1 [19,29-31]. Furthermore, we found that more than $90 \%$ of denatured DMMBP escapes out of the chaperonin cage of SRKKK2 mutant, whose three negatively charged residues (D359, D361, and E363) inside the cage are mutated to positively charged lysine. Thus, the SRKKK2-mediated folding in the above reports $[19,29,30]$ was in fact spontaneous folding [23]. To measure the in-cage folding mediated by SR1 and SRKKK2, urea, which does not induce above artifacts, should be used as the denaturant. 


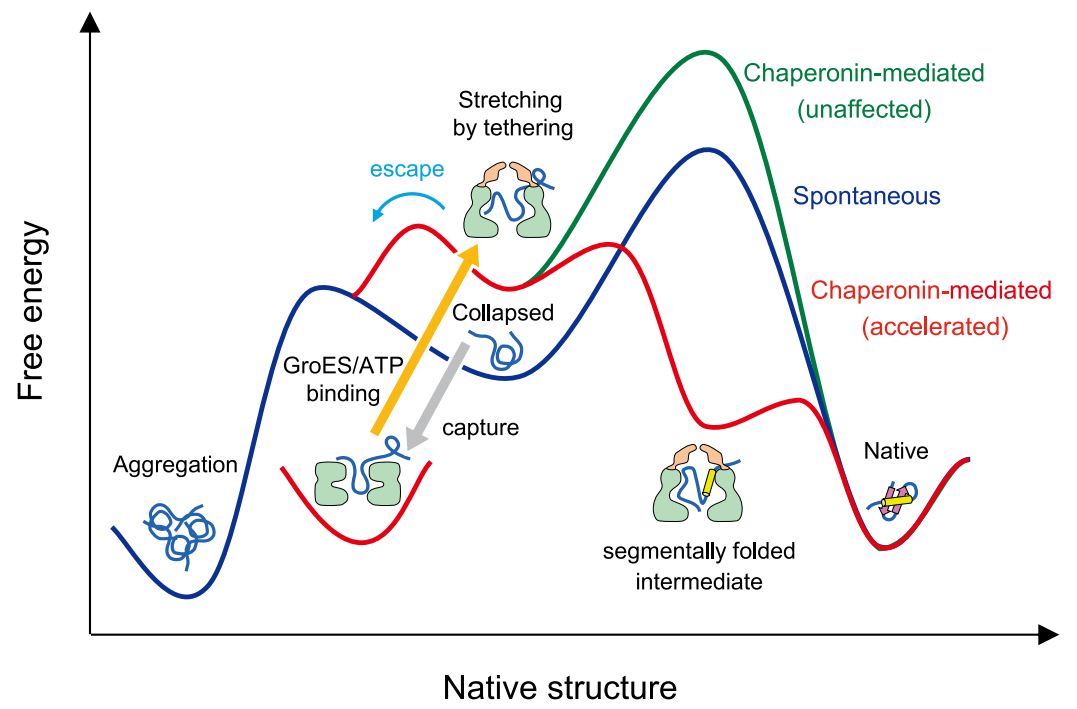

Figure 3 Hypothetical energy profile of chaperonin-mediated folding and spontaneous folding. Free energy is shown along the vertical axis. The fraction of native structure is shown along the horizontal axis. In spontaneous folding (blue lines), the denatured protein immediately forms a compact collapsed state containing many non-native hydrophobic interactions. A collapsed state folds through a pathway containing a large transition energy barrier (blue line). A collapsed state could form irreversible aggregates (left). The denatured protein is rapidly captured by GroEL. Upon GroES and ATP binding, the denatured protein is stretched by hydrophobic interactions (tethering) in the chaperonin cage. The tethered protein in the chaperonin cage could escape out of the cage. Secondary structures, especially $\alpha$-helices, form segmentally in the tethered protein, and subsequently assemble into the native protein, resulting in accelerated folding (red line). The tethered protein, which does not form segmental secondary structures, may fold in a spontaneous manner (green line).

\section{Discussions on the previous models}

The previously proposed models for the mechanism of chaperonin-mediated folding are still controversial. The arguments for each model are summarized below.

\section{Passive cage model}

In support of this model, it was reported that the rate of the chaperonin-mediated folding and that of the spontaneous folding of some proteins, such as wild-type MBP [29] and GFP [32], are almost the same at $25^{\circ} \mathrm{C}$. However, the chaperonin-mediated folding of rhodanese and mouse dihydrofolate reductase (DHFR) is slower than the spontaneous folding of these proteins $[20,33]$. For these substrate proteins, chaperonins seem to merely prevent the formation of aggregates, supporting the passive cage model. In contrast, it has been reported that the chaperonin-mediated folding of Rubisco is about four times faster than that of spontaneous folding at a low temperature [34,35]. Horwich and colleages suggested that the slow spontaneous folding of Rubisco can be attributed to the formation of small reversible aggregates [36]. However, using fluorescence cross-correlation spectroscopy (FCCS), Hartl and colleages showed that denatured DMMBP or dihydrodipicolinate synthase (DapA) does not form any aggregate, but chaperonins still accelerated the folding of these proteins [29,37]. Thus, the chaperonin cage does not always act passively in protein folding.

\section{Iterative annealing model}

This model proposed that the conformational changes in the chaperonin upon GroES binding induce a stretch-induced unfolding of denatured protein and the folding of denatured protein released inside the chaperonin cage. Incompletely folded proteins undergo further iterations until they achieve the native state. Lorimer and colleages proposed this model [38] and reported that the iterative folding by the chaperonin induces rapid amide proton-tritium exchange in Rubisco folding [39]. This model is also supported from simulation studies [40]. However, the rapid hydrogen exchange could not be reproduced by Horwich and colleages [24]. In addition, they reported that the amide proton-solvent deuterium exchange of mitochondrial malate dehydrogenase (MDH), a stringent substrate protein, does not increase upon encapsulation of denatured MDH into the chaperonin cage in contrast to the iterative annealing model [26]. Hartl and colleages reported that the folding rates of Rubisco mediated by SR1 and GroEL are almost the same, indicating that iterative annealing does not accelerate protein folding [35]. However, Rye and colleages recently proposed that forced unfolding of denatured Rubisco upon ATP binding and subsequent compaction of denatured Rubisco upon GroES binding accelerate Rubisco folding [41], and iterative annealing becomes effective at higher temperatures [42]. Although the effect of iterative annealing on Rubisco is still controversial, folding acceleration by iterative folding has not been observed in other proteins. We observed the coincidence of the folding rates mediated by SR1 and GroEL for DMMBP, even though 
about $90 \%$ of denatured DMMBP escaped out of the SR1 cage, suggesting that the recapture of denatured DMMBP by GroEL and the subsequent binding of GroES on the DMMBP-bound GroEL ring are very rapid and do not accelerate or decelerate the folding rate [23]. The turnover rate of chaperonins in the presence of the denatured protein is $\sim 10 \mathrm{~s}$ at $25^{\circ} \mathrm{C}$, and becomes shorter at higher temperatures [42,43]. The rapid turnover may help in maximizing the capacity of GroEL to capture up to two molecules of the denatured protein without reducing the folding rate, at the expense of ATP, but not in accelerating protein folding.

\section{Confinement model (Space restriction effect)}

The rapid decrease in the fluorescence anisotropy of denatured proteins upon GroES binding was reported and explained as the compaction of the denatured proteins, on the basis that florescence anisotropy reflects the radius of gyration of the labeled molecule and the fluorescence dye itself [44]. Observations of the conformation of Rubisco using fluorescence resonance energy transfer (FRET) showed that the distance of labeled fluorescence dyes becomes closer upon GroES binding than that before GroES binding [34]. Some simulation studies also proposed that the space restriction in the chaperonin cage accelerates protein folding, as the denatured protein is assumed to be repulsive to the chaperonin cage $[45,46]$. Supporting the simulation studies, Hartl and colleages reported that the decrease in the volume of the chaperonin cage by extension of the C-terminal peptide of GroEL induces accelerated folding of some proteins [29]. However, Horwich and colleages revisited their experiments and showed that the extension of the $\mathrm{C}$-terminal peptide does not result in folding acceleration [47].

Hartl and colleages also reported that the DMMBP mutant, containing two disulfide bonds, folds rapidly and is not accelerated by chaperonins, whereas the DMMBP mutant without disulfide bonds folds slowly and is accelerated by chaperonins [19]. Based on these results, it was proposed that conformational restrictions induced by disulfide bonds are similar to those induced by space restriction in the chaperonin cage [19]. However, since the disulfide bond in $\mathrm{N} 18 \mathrm{C} / \mathrm{D} 296 \mathrm{C}$ is in the core structure of the early folding intermediate, which is destabilized by the double mutations of V8G and Y283D, this disulfide bond could neutralize the destabilization effect [48]. The disulfide bond in D184C/ $\mathrm{K} 362 \mathrm{C}$ would stabilize the structure of the $\mathrm{C}$-terminal domain formed at the late folding step [48]. Therefore, the formation of two disulfide bonds could counteract the destabilization by the double mutations, and as a result, DMMBP mutant may behave like wild-type MBP, the folding of which is not accelerated by chaperonins. Recently, Hartl and colleages showed that the folding of DapA $(31 \mathrm{kDa})$ is accelerated by approximately 30 -folds by chaperonins. Since the molecular weight of DapA is smaller than that of molecules that were efficiently accelerated by chaperonins in the simulation studies $[45,46]$, the acceleration of folding by chaperonins may not be explained solely by the space restriction effect.

It is also proposed that space restriction is induced by structured water molecules bound on the surface of the chaperonin cage. Chakraborty et al. reported that the negatively charged residues, which are mutated in SRKKK2, and osmolyte trimethylamineoxide (TMAO) similarly contribute to form water structures on the surface of the chaperonin cage [19]. This hypothesis relies on the fact that the rates of SRKKK2-mediated folding and spontaneous folding are the same at various TMAO concentrations. However, the SRKKK2-mediated folding measured in this study turned out to be spontaneous folding out of the SRKKK2 cage, as described above. Moreover, the measurement of the correct in-cage folding with increasing concentrations of TMAO reveals that the SRKKK2-mediated folding is not accelerated by TMAO, but the SR1-mediated folding and the spontaneous folding are accelerated, suggesting that the mechanism of spontaneous folding and that of SRKKK2mediated folding are different, even though their folding rates are similarly slow [23].

\section{Hydrophobic interactions in the chaperonin cage}

All models aside from the tethering model suppose that the interaction between the denatured protein and the chaperonin cage wall is repulsive or ineffective. However, several observations suggest that the interaction of the denatured protein with the chaperonin cage wall affects chaperonin-mediated folding. The mutation of Y71 of GroES, which is exposed inside the chaperonin cage (Fig. 1B) to hydrophilic residues, results in the increase in GFP fluorescence in vivo [49]. We reported that Y203 of SR1 at the hydrophobic interface of the chaperonin cage is important both in accelerating the protein folding and in preventing the escape of the denatured protein from the cage [20,23]. It has been shown that the depletion of hydrophobic C-terminal peptides of GroEL, the largest hydrophobic portion in the chaperonin cage, results in slower folding [29,30,50,51]. On the contrary, the increase in hydrophobicity in the chaperonin cage, caused by the chemical labeling of pyrene maleimide, decreases the rate of protein folding [20,52]. The interaction between denatured Rubisco and the C-terminal peptides of the GroEL(S43C) mutant labeled with pyrene has been observed by cryo-electron microscopy [53]. However, it is possible that hydrophobic pyrene, labeled at the bottom of the chaperonin cage, induces excess interactions to the $\mathrm{C}$-terminal peptides. Thus, the number of hydrophobic residues and their positions in the chaperonin cage would be important for the balance between protein folding acceleration and the retention of denatured proteins in the chaperonin cage.

The effect of hydrophobic interactions has also been proposed by simulation studies. Jewett et al. reported that weak hydrophobic interactions between the folding intermediate and the chaperonin cage wall allow iterative denaturation 
and folding without iterative cycling by ATP hydrolysis, provide another folding pathway, and decrease the transition energy barrier for protein folding using the coarse-grained molecular simulation of small model peptide [54,55]. The denatured protein in the chaperonin cage has higher energy than that in spontaneous folding, as suggested by Jewett et $a l$, since the denatured protein in the chaperonin cage can intrinsically escape out of the cage and fold spontaneously (Fig. 3) [20,23]. It is known that in the spontaneous folding of large proteins, the proteins initially form non-native hydrophobic interactions (hydrophobic collapse) [48,56-58]. If stable non-native hydrophobic interactions inhibit spontaneous folding, the destabilization of non-native hydrophobic interactions in the chaperonin would result in the acceleration of the protein folding rate. Recently, Sirur et al. reported the coarse-grained molecular simulation of rhodanese folding in the chaperonin cage [59]. They showed that when rhodanese-chaperonin interaction is included in the simulation, rhodanese folds more slowly in the chaperonin cage than it does in spontaneous folding as reported experimentally [20,33], but when the interaction is repulsive, it folds one to two orders of magnitude faster than it does in spontaneous folding. This observation suggests that the intermolecular interaction is strong enough to offset the space restriction effect. The protrusion of denatured rhodanese out of the chaperonin cage is observed in this simulation when intermolecular interaction is included. However, polypeptide protrusion from the hydrophobic interface observed in our study [20] is not reproduced, since the conformational fluctuations of the chaperonin subunits are not calculated in this simulation.

\section{Protein folding trajectory in the chaperonin cage}

To know how chaperonins accelerate protein folding, protein folding trajectories should be compared between chaperonin-mediated folding and spontaneous folding. Since many stringent substrate proteins easily form aggregates during spontaneous folding, the measurement of the folding trajectory in spontaneous folding is difficult, and only the early intermediate of spontaneous folding can be measured when the denatured protein is diluted in the buffer medium with the usual method. Lin et al. measured the trajectory of Rubisco folding using FRET [34]. They showed that the stretched denatured Rubisco bound on GroEL is compacted upon GroES binding and is subsequently extended by folding into its native state. In the initial spontaneous folding, the denatured Rubisco formed a more compact state than that in chaperonin-mediated folding. Hofmann et al. measured the chaperonin-mediated folding and spontaneous folding of rhodanese by FRET using confocal single molecule fluorescence spectroscopy to observe single-molecular fluorescence of rhodanese exclusively from its aggregates [33]. In the chaperonin cage, the C-terminal domain of rhodanese folds more slowly than it does in spontaneous folding, but the initial conformation of the folding does not change significantly. Horst et al. measured the folding trajectory of human DHFR refolding using amide proton-solvent deuterium exchange monitored by NMR [25]. The protection of amide proton exchange is not largely altered by chaperonins. Since chaperonins do not greatly accelerate the folding rate of human DHFR, it was concluded that DHFR folding is not largely affected by chaperonins except in its folding yield. Park et al. measured the time-course of disulfide bond formation in trypsinogen by LC-MS analysis [60]. Denatured trypsinogen in the chaperonin cage initially forms shortrange disulfides in the primary sequence, similar to that in spontaneous folding in bulk medium, but at the latter folding step, disulfides are restructured and long-range disulfides are formed in the native state [60]. The fact that chaperonins do not change the initial conformation of the denatured protein significantly supports the passive model.

Recently, Georgescauld et al. measured the folding trajectory of DapA, an obligate substrate protein that requires chaperonins to fold in vivo $[61,62]$, by amide proton-solvent deuterium exchange pulse-labeling monitored with LC-MS analysis (HX-MS) [37]. The chaperonin-mediated folding of DapA is 30 -fold faster than its spontaneous folding. The extent of folding acceleration of DapA is the highest among the values reported so far. In the spontaneous folding of DapA, most of the structures in the TIM-barrel domain form slowly at once. In contrast, in the chaperonin-mediated folding, a set of $\alpha$-helices in the TIM-barrel domain forms rapidly and subsequently, $\beta$-sheets and other structures are formed. This type of folding was named as segmental folding. These results imply that the chaperonin cage enables $\alpha$-helices to form at the early folding step, and long-distance interactions between $\beta$-sheets are formed at the latter folding step. It is known that $\alpha$-helices can form immediately at the early stage of folding via the formation of hydrogen bonds within adjacent amino acids [58]. Interestingly, the amide protons in one $\alpha$-helix of the denatured DapA are protected, indicating that this region forms $\alpha$-helices from the beginning or strongly interacts with the hydrophobic apical surface of GroEL. The protection of the amide proton in the denatured protein bound on GroEL is also observed for $\mathrm{MDH}$, whose folding is greatly accelerated by chaperonin [26]. It is possible that the hydrophobic surface in the chaperonin cage stabilizes and promotes the formation of amphipathic $\alpha$-helices as shown for the model peptide [63]. However, these early structures are unstable and collapse easily in bulk medium, since denatured proteins released from GroEL by ATP hydrolysis without GroES cannot fold rapidly. Thus, after the denatured protein is released into the chaperonin cage, the early structures would be continuously stabilized by hydrophobic interactions in the chaperonin cage. The space restriction effect may contribute the formation of $\beta$-sheets, which are distant in the amino-acid sequence. 


\section{Summary}

Although it has been considered that the interaction between denatured proteins and the chaperonin cage wall does not largely affect protein folding at first, recent results of experimental and simulation studies have suggested that the hydrophobic interactions affect chaperonin-mediated folding. As described above, the mutations in the hydrophobic residues of GroEL decrease the folding rate [20,29,30,50,51]. These hydrophobic residues are also important in retaining the denatured protein inside the cage, but the excess hydrophobic surfaces decelerate protein folding [20]. The chaperonin cage intrinsically allows denatured proteins to escape out of the cage, as observed for DMMBP [23]. If no hydrophobic residues are exposed in the chaperonin cage, most of denatured protein could have escaped out of the cage and the chaperonin would not be able to assist protein folding. Thus, the extent of hydrophobicity in the chaperonin cage would be evolutionally determined by the balance between the retention of the denatured protein and the effect on protein folding rate. The loss of escaped denatured protein by aggregate formation is suppressed by the rapid recapture to other GroEL molecule and subsequent chaperonin-mediated folding without the actual decrease of folding rate.

It becomes apparent that the extent of folding acceleration by chaperonins is largely dependent on the substrate proteins $[29,34,35,37]$. Protein folding is not always accelerated and even decelerated in the chaperonin cage. The chaperonin cage could be effective to accelerate the folding of a set of structural motifs found in obligate substrate proteins, such as the TIM-barrel motif in DapA and Rubisco [4,61,62], but other examples should be demonstrated. The extent of folding acceleration may be dependent on the importance of segmental folding in each protein (Fig. 3). However, it is still possible that the spontaneous folding of DapA is stacked in an initial hydrophobic collapsed state, and folds through a similar folding trajectory as that in chaperonin-mediated folding. If this is true, chaperonins do not accelerate protein folding by allowing segmental folding, but destabilize the collapsed state to allow iterative denaturation and folding as suggested from simulation study [54,55].

The recent results suggest that the hydrophobic interactions in the chaperonin cage could contribute to the chaperonin-mediated folding for the retention of the denatured protein in the restricted space, the destabilization of the non-native hydrophobic interactions formed in a collapsed state of the denatured protein, and the facilitation of segmental structure formation. The combination of the restricted space effect and the hydrophobic interactions in the chaperonin cage would result in the iterative restructuring of denatured protein. Thus, the factors assumed in all the proposed models seem to contribute synergistically in the chaperonin cage. However, these effects on protein folding would be largely dependent on the structural and folding properties of protein. It is still unclear how chaperonins change protein folding and why the folding of some proteins is greatly accelerated, but that of other proteins is not. Further experiments and simulation studies will provide new findings to answer these questions, and the controversy on the folding mechanism of chaperonins that has persisted for a quartercentury might be settled in the near future.

\section{Acknowledgements}

I thank Missaukee Yoshida for helpful suggestions and comments. I acknowledge financial supports for our studies by the Ministry of Education, Culture, Sports, Science \& Technology (MEXT)-Japan Society for Promotion of Science (JSPS) Grants-in-Aid for Scientific Research and by funds from the Private University Strategic Research Foundation Support Program from MEXT.

\section{References}

[1] Anfinsen, C. B. Principles that govern the folding of protein chains. Science 181, 223-230 (1973).

[2] Tissières, A., Mitchell, H. K. \& Tracy, U. M. Protein synthesis in salivary glands of Drosophila melanogaster: relation to chromosome puffs. J. Mol. Biol. 84, 389-398 (1974).

[3] Cheng, M. Y., Hartl, F. U., Martin, J., Pollock, R. A., Kalousek, F., Neupert, W., et al. Mitochondrial heat-shock protein hsp60 is essential for assembly of proteins imported into yeast mitochondria. Nature 337, 620-625 (1989).

[4] Goloubinoff, P., Christeller, J. T., Gatenby, A. A. \& Lorimer, G. H. Reconstitution of active dimeric ribulose biphosphate carboxylase from an unfolded state depends on two chaperonin proteins and Mg-ATP. Nature 342, 884-889 (1989).

[5] Hemmingsen, S. M., Woolford, C., Vies, S. M. van der, Tilly, K., Dennis, D. T., Georgopoulos, C. P., et al. Homologous plant and bacterial proteins chaperone oligomeric protein assembly. Nature 333, 330-334 (1988).

[6] Goloubinoff, P., Gatenby, A. A. \& Lorimer, G. H. GroE heatshock proteins promote assembly of foreign prokaryotic ribulose bisphosphate carboxylase oligomers in Escherichia coli. Nature 337, 44-47 (1989).

[7] Bukau, B., Weissman, J. \& Horwich, A. Molecular chaperones and protein quality control. Cell 125, 443-451 (2006).

[8] Fan, C.-Y., Lee, S. \& Cyr, D. M. Mechanisms for regulation of Hsp70 function by Hsp40. Cell Stress Chaperones 8, 309-316 (2003).

[9] Braig, K., Otwinowski, Z., Hegde, R., Boisvert, D. C., Joachimiak, A., Horwich, A. L., et al. The crystal structure of the bacterial chaperonin GroEL at 2.8 A. Nature 371, 578-586 (1994).

[10] Fenton, W. A., Kashi, Y., Furtak, K. \& Horwich, A. L. Residues in chaperonin GroEL required for polypeptide binding and release. Nature 371, 614-619 (1994).

[11] Xu, Z., Horwich, A. L. \& Sigler, P. B. The crystal structure of the asymmetric GroEL-GroES-(ADP)7 chaperonin complex. Nature 388, 741-750 (1997)

[12] Todd, M. J., Viitanen, P. V. \& Lorimer, G. H. Dynamics of the chaperonin ATPase cycle: implications for facilitated protein folding. Science 265, 659-666 (1994).

[13] Koike-Takeshita, A., Yoshida, M. \& Taguchi, H. Revisiting the GroEL-GroES reaction cycle via the symmetric intermediate implied by novel aspects of the GroEL(D398A) mutant. $J$. Biol. Chem. 283, 23774-23781 (2008). 
[14] Sameshima, T., Iizuka, R., Ueno, T., Wada, J., Aoki, M., Shimamoto, N., et al. Single-molecule study on the decay process of the football-shaped GroEL-GroES complex using zero-mode waveguides. J. Biol. Chem. 285, 23159-23164 (2010).

[15] Sameshima, T., Iizuka, R., Ueno, T. \& Funatsu, T. Denatured proteins facilitate the formation of the football-shaped GroEL-(GroES)2 complex. Biochem. J. 427, 247-254 (2010).

[16] Yang, D., Ye, X. \& Lorimer, G. H. Symmetric GroEL: GroES2 complexes are the protein-folding functional form of the chaperonin nanomachine. Proc. Natl. Acad. Sci. USA 110, E42984305 (2013).

[17] Ye, X. \& Lorimer, G. H. Substrate protein switches GroE chaperonins from asymmetric to symmetric cycling by catalyzing nucleotide exchange. Proc. Natl. Acad. Sci. USA 110, E4289-4297 (2013).

[18] Mendoza, J. A., Rogers, E., Lorimer, G. H. \& Horowitz, P. M. Chaperonins facilitate the in vitro folding of monomeric mitochondrial rhodanese. J. Biol. Chem. 266, 13044-13049 (1991).

[19] Chakraborty, K., Chatila, M., Sinha, J., Shi, Q., Poschner, B. C., Sikor, M., et al. Chaperonin-catalyzed rescue of kinetically trapped states in protein folding. Cell 142, 112-122 (2010).

[20] Motojima, F. \& Yoshida, M. Polypeptide in the chaperonin cage partly protrudes out and then folds inside or escapes outside. EMBO J. 29, 4008-4019 (2010).

[21] Weissman, J. S., Hohl, C. M., Kovalenko, O., Kashi, Y., Chen, S., Braig, K., et al. Mechanism of GroEL action: productive release of polypeptide from a sequestered position under GroES. Cell 83, 577-587 (1995).

[22] Weissman, J. S., Rye, H. S., Fenton, W. A., Beechem, J. M. \& Horwich, A. L. Characterization of the active intermediate of a GroEL-GroES-mediated protein folding reaction. Cell $\mathbf{8 4}$, 481-490 (1996).

[23] Motojima, F., Motojima-Miyazaki, Y. \& Yoshida, M. Revisiting the contribution of negative charges on the chaperonin cage wall to the acceleration of protein folding. Proc. Natl. Acad. Sci. USA 109, 15740-15745 (2012).

[24] Park, E. S., Fenton, W. A. \& Horwich, A. L. No evidence for a forced-unfolding mechanism during ATP/GroES binding to substrate-bound GroEL: no observable protection of metastable Rubisco intermediate or GroEL-bound Rubisco from tritium exchange. FEBS Lett. 579, 1183-1186 (2005).

[25] Horst, R., Fenton, W. A., Englander, S. W., Wüthrich, K. \& Horwich, A. L. Folding trajectories of human dihydrofolate reductase inside the GroEL GroES chaperonin cavity and free in solution. Proc. Natl. Acad. Sci. USA 104, 20788-20792 (2007).

[26] Chen, J., Walter, S., Horwich, A. L. \& Smith, D. L. Folding of malate dehydrogenase inside the GroEL-GroES cavity. Nat. Struct. Biol. 8, 721-728 (2001).

[27] Nojima, T., Murayama, S., Yoshida, M. \& Motojima, F. Determination of the number of active GroES subunits in the fused heptamer GroES required for interactions with GroEL. J. Biol. Chem. 283, 18385-18392 (2008).

[28] Farr, G. W., Furtak, K., Rowland, M. B., Ranson, N. A., Saibil, H. R., Kirchhausen, T., et al. Multivalent binding of nonnative substrate proteins by the chaperonin GroEL. Cell 100, 561$573(2000)$.

[29] Tang, Y.-C. C., Chang, H.-C. C., Roeben, A., Wischnewski, D., Wischnewski, N., Kerner, M. J., et al. Structural features of the GroEL-GroES nano-cage required for rapid folding of encapsulated protein. Cell 125, 903-914 (2006).

[30] Tang, Y. C., Chang, H. C., Chakraborty, K., Hartl, F. U. \& Hayer-Hartl, M. Essential role of the chaperonin folding compartment in vivo. EMBO J. 27, 1458-1468 (2008).
[31] Sharma, S., Chakraborty, K., Muller, B. K., Astola, N., Tang, Y.C., Lamb, D. C., et al. Monitoring protein conformation along the pathway of chaperonin-assisted folding. Cell 133, 142-153 (2008).

[32] Makino, Y., Amada, K., Taguchi, H. \& Yoshida, M. Chaperoninmediated folding of green fluorescent protein. J. Biol. Chem. 272, 12468-12474 (1997).

[33] Hofmann, H., Hillger, F., Pfeil, S. H., Hoffmann, A., Streich, D., Haenni, D., et al. Single-molecule spectroscopy of protein folding in a chaperonin cage. Proc. Natl. Acad. Sci. USA 107, 11793-11798 (2010).

[34] Lin, Z. \& Rye, H. S. Expansion and compression of a protein folding intermediate by GroEL. Mol. Cell 16, 23-34 (2004).

[35] Brinker, A., Pfeifer, G., Kerner, M. J., Naylor, D. J., Hartl, F. U. \& Hayer-Hartl, M. Dual function of protein confinement in chaperonin-assisted protein folding. Cell 107, 223-233 (2001).

[36] Apetri, A. C. \& Horwich, A. L. Chaperonin chamber accelerates protein folding through passive action of preventing aggregation. Proc. Natl. Acad. Sci. USA 105, 17351-17355 (2008).

[37] Georgescauld, F., Popova, K., Gupta, A. J., Bracher, A., Engen, J. R., Hayer-Hartl, M., et al. GroEL/ES Chaperonin Modulates the Mechanism and Accelerates the Rate of TIM-Barrel Domain Folding. Cell 157, 922-934 (2014).

[38] Todd, M. J., Lorimer, G. H. \& Thirumalai, D. Chaperoninfacilitated protein folding: optimization of rate and yield by an iterative annealing mechanism. Proc. Natl. Acad. Sci. USA 93, 4030-4035 (1996).

[39] Shtilerman, M., Lorimer, G. H. \& Englander, S. W. Chaperonin function: folding by forced unfolding. Science 284, 822 825 (1999).

[40] Betancourt, M. R. \& Thirumalai, D. Exploring the kinetic requirements for enhancement of protein folding rates in the GroEL cavity. J. Mol. Biol. 287, 627-644 (1999).

[41] Lin, Z., Madan, D. \& Rye, H. S. GroEL stimulates protein folding through forced unfolding. Nat. Struct. Mol. Biol. 15, 303-311 (2008).

[42] Lin, Z., Puchalla, J., Shoup, D. \& Rye, H. S. Repetitive protein unfolding by the trans ring of the GroEL-GroES chaperonin complex stimulates folding. J. Biol. Chem. 288, 30944-30955 (2013).

[43] Mendoza, J. A., Warren, T. \& Dulin, P. The ATPase activity of chaperonin GroEL is highly stimulated at elevated temperatures. Biochem. Biophys. Res. Commun. 229, 271-274 (1996).

[44] Rye, H. S., Burston, S. G., Fenton, W. A., Beechem, J. M., Xu, Z., Sigler, P. B., et al. Distinct actions of cis and trans ATP within the double ring of the chaperonin GroEL. Nature $\mathbf{3 8 8}$ 792-798 (1997).

[45] Baumketner, A., Jewett, A. \& Shea, J. E. Effects of confinement in chaperonin assisted protein folding: rate enhancement by decreasing the roughness of the folding energy landscape. J. Mol. Biol. 332, 701-713 (2003).

[46] Takagi, F., Koga, N. \& Takada, S. How protein thermodynamics and folding mechanisms are altered by the chaperonin cage: molecular simulations. Proc. Natl. Acad. Sci. USA 100, 11367-11372 (2003).

[47] Farr, G. W., Fenton, W. A. \& Horwich, A. L. Perturbed ATPase activity and not "close confinement" of substrate in the cis cavity affects rates of folding by tail-multiplied GroEL. Proc. Natl. Acad. Sci. USA 104, 5342-5347 (2007).

[48] Walters, B. T., Mayne, L., Hinshaw, J. R., Sosnick, T. R. \& Englander, S. W. Folding of a large protein at high structural resolution. Proc. Natl. Acad. Sci. USA 110, 18898-18903 (2013).

[49] Wang, J.D., Herman, C., Tipton, K.A., Gross, C. A. \& 
Weissman, J.S. Directed evolution of substrate-optimized GroEL/S chaperonins. Cell 111, 1027-1039 (2002).

[50] Machida, K., Kono-Okada, A., Hongo, K., Mizobata, T. \& Kawata, Y. Hydrophilic residues 526 KNDAAD 531 in the flexible C-terminal region of the chaperonin GroEL are critical for substrate protein folding within the central cavity. $J$. Biol. Chem. 283, 6886-6896 (2008).

[51] Weaver, J. \& Rye, H. S. The C-terminal tails of the bacterial chaperonin GroEL stimulate protein folding by directly altering the conformation of a substrate protein. J. Biol. Chem. 289, 23219-23232 (2014).

[52] Madan, D., Lin, Z. \& Rye, H. S. Triggering protein folding within the GroEL-GroES complex. J. Biol. Chem. 283, 3200332013 (2008).

[53] Chen, D.-H., Madan, D., Weaver, J., Lin, Z., Schröder, G. F., Chiu, W., et al. Visualizing GroEL/ES in the act of encapsulating a folding protein. Cell 153, 1354-1365 (2013).

[54] Jewett, A. I., Baumketner, A. \& Shea, J. E. Accelerated folding in the weak hydrophobic environment of a chaperonin cavity: creation of an alternate fast folding pathway. Proc. Natl. Acad. Sci. USA 101, 13192-13197 (2004).

[55] Jewett, A. I. \& Shea, J.E. Folding on the chaperone: yield enhancement through loose binding. J. Mol. Biol. 363, 945957 (2006).

[56] Agashe, V. R., Shastry, M. C. \& Udgaonkar, J. B. Initial hydro- phobic collapse in the folding of barstar. Nature 377, 754-757 (1995).

[57] Daggett, V. \& Fersht, A. R. Is there a unifying mechanism for protein folding? Trends Biochem. Sci. 28, 18-25 (2003).

[58] Dobson, C. M. \& Karplus, M. The fundamentals of protein folding: bringing together theory and experiment. Curr. Opin. Struct. Biol. 9, 92-101 (1999).

[59] Sirur, A. \& Best, R. B. Effects of interactions with the GroEL cavity on protein folding rates. Biophys. J. 104, 1098-1106 (2013).

[60] Park, E. S., Fenton, W. A. \& Horwich, A. L. Disulfide formation as a probe of folding in GroEL-GroES reveals correct formation of long-range bonds and editing of incorrect shortrange ones. Proc. Natl. Acad. Sci. USA 104, 2145-2150 (2007).

[61] Kerner, M. J., Naylor, D. J., Ishihama, Y., Maier, T., Chang, H.-C., Stines, A. P., et al. Proteome-wide analysis of chaperonindependent protein folding in Escherichia coli. Cell 122, 209 220 (2005).

[62] Fujiwara, K., Ishihama, Y., Nakahigashi, K., Soga, T. \& Taguchi, H. A systematic survey of in vivo obligate chaperonindependent substrates. EMBO J. 29, 1552-1564 (2010).

[63] Li, Y., Gao, X. \& Chen, L. GroEL recognizes an amphipathic helix and binds to the hydrophobic side. J. Biol. Chem. 284, 4324-4331 (2009). 